\title{
Hydroponics Checking and Control Framework: An Iot based Methodology: A Review
}

\section{S.R. Balaji, K. Santhosh}

Bannari Amman Institute of Technology, Sathyamangalam, Erode-638 401, Tamil Nadu, India.

Received: August 2021

Accepted: August 2021

\section{ABSTRACT}

Web of Things (loT) is the quickly creating fields for giving social and monetary focal points for rising and making an economy of the country. loT field is prospering in regions like clinical, horticulture, transportation, preparing and So forth This is of maximum importance because hydroponics is a retrogressive locale of carried out science. Diverged from different zones like agribusiness, thus, it's fundamental to decide the issues that are around here with the help of innovation. Water quality may be an essential issue, it fundamentally relies on various boundaries like broke up oxygen, carbonates, turbidity, alkali, nitrates, salt, $\mathrm{pH}$, temperature and so forth. The proposed framework consistently screens the water superb boundary the usage of sensors, the incredible information is exceeded immediately to the water rancher bendy via the cloud. Among the issues, the sluggish dormant period inside the consideration of water quality and along these lines the wastage of assets like water, in development are the important issues that must be tended to. It is used to utilizations the wastewater from the aquarium to develop the plants, thus, the $\mathrm{pH}$ and alkali killed water from hydrogen dust pellets in broaden mattress is looked after lower back to the aquarium.

Key words: Hydroponics, Machine to machine (M2M) connectivity, Round trip time (RTT).

Hydroponics is one of the thriving fragments in agricultural nations like India as it contributes 1.07 percent of the GDP. It is discovered that fish need of the country by 2025 would be as far as 1.6 crores tones and because of the overfishing, customary fisheries have been depleted in this manner business hydroponics has been showing up. Hydroponics includes the course of action of activities, data and strategies for the raising of submerged plants and a couple of kinds of creatures in the water. This activity has inconceivable importance in financial progression and food advancement. Consistent checking of the physical, engineered and natural rule of lake or lake water helps not exclusively to recognize and manage the horrible conditions of hydroponics however furthermore to preserve a separation from ordinary damage and the breakdown of the revolutionary interaction. The seeing of physical and substance factors like $\mathrm{pH}$, oxygen and temperature in the water is pivotal to keep up adequate conditions and stay away from disastrous conditions that cause the disappointment of hydroponics. Hydroponics, known as aquafarming, is the cultivating of sea-going animals, for instance, foragers, fish and crabs. The proposed work upholds far off seeing of the fish cultivating reliant upon Internet of Things (IoT) for progressing checking, control of a fish cultivating and the significant issue like wastage of water in hydroponics are controlled with hydroponics, additionally called the coordination of aqua-farming with hydroponics, has created to be a productive model of possible regular and natural food creation. The agreeable association between fish, plants and minute life forms, in a controlled space, depends on ideal water quality conditions. This requires a need to make steady water quality checking techniques that rely upon sharp data getting, correspondence and taking care of. This work revolves around using the Internet of Things (loT) innovation to screen and control water quality boundaries using sensors that give far off, determined and consistent information of pointers identified with water quality, on a graphical client interface(GUI). A planned work containing a Raspberry Pi 3 and business sensor circuits and tests that action $\mathrm{pH}$, water temperature and turbidity were passed on in an aquarium and the information acquired from the sensors is moved to ThingSpeak, an loT examination stage administration that gives consistent informationAquaculture is one of the flourishing portions in non-industrial nations like India as it contributes 1.07 percent of the GDP. It is discovered that fish need of the country by 2025 would be as far as 1.6 crores tones and because of the overfishing, customary fisheries have been depleted subsequently business hydroponics has been showing up. Hydroponics includes the course of action of activities, data and strategies for the raising of submerged plants and a couple of kinds of creatures in the water. This activity has amazing importance in money-related progression and food advancement. Consistent checking of the physical, manufactured and natural rule of lake or lake water helps not exclusively to distinguish and control the horrific situations of hydroponics but furthermore to preserve a separation from normal harm and the breakdown of the advent cycle. The seeing of

*Corresponding author's E-mail: santhosh.ag19@bitsathy.ac.in 
physical and substance factors like $\mathrm{pH}$, oxygen and temperature in the water is vital to keep up adequate conditions and stay away from appalling conditions that cause the disappointment of hydroponics. Hydroponics, known as aquafarming, is the cultivation of amphibian animals, for instance, scroungers, fish and crabs. The proposed work upholds distant seeing of the fish cultivating reliant upon Internet of Things (IoT) for continuous checking, control of a fish cultivating and the difficult issue like wastage of water in hydroponics are controlled with hydroponics, additionally called the coordination of aqua-farming with hydroponics, has created to be a productive model of plausible characteristic and natural food creation. The agreeable association between fish, plants and minuscule creatures, in a controlled space, depends on ideal water quality conditions. This requires a need to make steady water quality checking methods that rely upon sharp data getting, correspondence and dealing with. This work revolves around using the Internet of Things (loT) innovation to screen and control water quality boundaries using sensors that give distant, constant and persistent information of pointers identified with water quality, on a graphical client interface(GUI). A planned work containing a Raspberry Pi 3 and business sensor circuits and tests that action $\mathrm{pH}$, water temperature and turbidity were passed on in an aquarium and the information acquired from the sensors is moved to ThingSpeak, an loT examination stage administration that gives persistent data portrayal and assessment. Reliable seeing of this data and making fundamental adjustments, will support the upkeep of a sound climate this is useful for the development of fish and plant life even as making use of around $90 \%$ much less water than ordinary cultivating.

Business hydroponics is confronting various difficulties on account of unexpected natural condition varieties that end up in changes in water quality boundaries. As of now, water ranchers utilize manual check techniques for knowing the boundaries of water. This will take longer and is not right since water quality boundaries could change regarding time. To stay away from this drawback, development ought to be engaged with hydroponics that improves the intensity and limits the misfortunes by steady checking of water quality boundaries. The objective of this venture is to plan and execute a circulated framework for hydroponics water quality consideration through far-off seeing of turbidity, temperature and $\mathrm{pH}$. This work will contribute far off checking structure through loT to screen water quality in lakes. The framework is convenient, secluded, minimal expense, adaptable and licenses sharing of information through the cloud that can be utilized for the progression and improvement of hydroponics-related exercises.

\section{Related works}

A few articles use Arduino as a little regulator for watching the water field Raju and Verma (2017), in any case, Raspberry $\mathrm{Pi}-3$ is significantly in addition superior contrasted and Arduino because it has an inborn Wi-Fi module. A few papers center around hardly any benevolent sensors like turbidity, DO, pH, Chandanapalli et al (2014), Simbeye et al (2014) Deng et al (2010) so forth and an answer for those issues. Be that as it may, the development of sea-going life relies upon a few imperatives like Ammonia, Carbonates, Nitrate, Bi-Carbonates, Salt and so forth All the above boundaries are estimated with the assistance of a few sensors and a potential arrangement was given to the water rancher to keep up the lake. The constantly recognized data is sent straightforwardly to the water rancher through application Encinas et al (2017) Hongpin et al (2015) and Nocheski and Naumoski (2018). As of late loT is arriving at a high level with its application to ranchers Chavan et al (2018) and Preetham et al. (2019). numerous papers in writing study center around how the amphibian life gets occupied because of adjustment in water quality boundaries regarding time and how loT innovation is utilized to conquer the issues haron et al (2008) Israni et al (2015) Preetham et al (2019). In addition, sending and putting away the data in the cloud helps the client for investigating the information by information examination, which may work with us to take support of dynamic measures before the change in water quality boundaries Chen et al (2015). the above-planned framework is invigorated with the help of city power with battery. In any case, aquafarmers face power slices and this issue must be tended to Preetham et al (2019). Our proposed model will answer the water rancher before the harm was done to the oceanic animals and aquafarmer gets an alarm message if the limits of the water lake surpass the predetermined restricted range.

\section{IoT based aquaculture monitoring and control system}

The proposed model overwhelmingly focuses on constantly noticing the water quality factors at unsurpassed to make preventive strides right on time to hurt water creatures. The proposed design has 4 sections, (1) power module, (2) sensor module, (3) regulator module, (4) yield module. The point-by-point block graph of the structure is in Fig 1.

\section{System features}

The work is worried about three significant issues Effective estimation of water quality boundaries, Monitoring, Control and Ease of access.

\section{(a) Effective estimation of water quality boundaries}

The task has a sensor module that incorporates a couple of sensors, for instance, Turbidity, $\mathrm{pH}$, Temperature, Salt, These sensors are fixed on Raspberry $\mathrm{Pi}$ and are utilized for recognizing the water boundaries constantly.

\section{(b) Monitoring}

The sensor information has been observed utilizing the thing talk cloud.

\section{(c) Control}

Based on the sensor information and limit esteems the water lake can be controlled. 


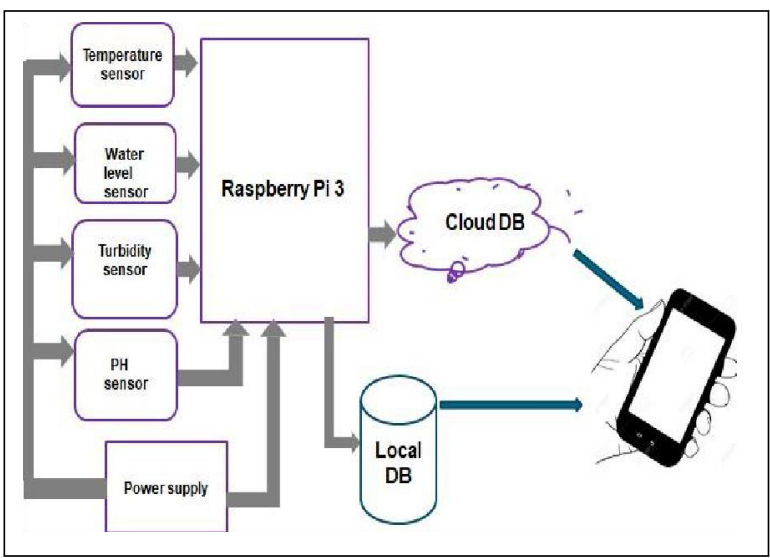

Fig 1: Block Diagram of IAMCS.

\section{(d) Ease of access}

The information can be adequately gotten to utilizing the cloud and it very well may be noticed and controlled utilizing the versatile application.

\section{Block diagram description}

\section{Power module}

The force module has a DC-DC converter, charge regulator, battery. The battery is transcendently used to supply control in the night as water quality boundaries generally change around evening time. A DC-DC converter is there to enable to scale regulator module which will work at $5 \mathrm{~V}$. A DC-DC converter is mostly used to give a constant voltage.

\section{Sensor module}

The sensor module comprises specific sensors, for instance, $\mathrm{pH}$, turbidity, water level, Temperature. These sensors are associated with Raspberry $\mathrm{Pi}$ and are utilized for distinguishing the water boundaries occasionally.

\section{Controller module}

It is treated as the main piece of this undertaking. Raspberry $\mathrm{Pi}-3$ model $\mathrm{B}$ is utilized as a regulator. Raspberry $\mathrm{Pi}$ is a low spending plan, a little PC board with Linux as a functioning system. It has an enormous number of good conditions when stood out from other limited scope regulators, for instance, the inbuilt Wi-Fi module. The Program for getting the sensor data is written in the python language and sent that data to the cloud. The worker side program continually spectators the sensor regards whether they are inside the edge expand. If the characteristics go wrong from the edge to expand, a telling message with the plan is shipped off the versatile application that is a yield module.

\section{Output module}

Aquafarmer versatile is treated as a yield area. An application has been created in the cell phone which has a few gadgets to show the sensor information and different catches to control the progression of water through the engine and if the detected facts surpass the restrict levels geared up Message can be shipped off the rancher with vital strides to be taken.

\section{Implementation}

The execution of the whole framework is classified as far as Hardware and Software. When the equipment part is amassed that is sensor hubs with Raspberry $\mathrm{Pi}$, comes the product part. Execution is done mostly in three distinct spaces - Python, Cloud and Android. ThingSpeak is utilized for executing cloud tasks. Android App is created utilizing Android Studio. Raspberry Pi utilizes Raspbian as an Operating System (OS) and Python IDLE is utilized for composing Python codes. What's more, Putty and VNC servers are likewise utilized for getting to the Raspberry $\mathrm{Pi}$ terminal from a PC without associating Raspberry Pi to display screen and separate console and mouse. The correspondence system between various hubs depends on I2C or SPI convention. SPI (Serial fringe interface) is an availability convention for the machine-to-machine (M2M) correspondence. It was planned as transportation of incredibly lightweight informing and distributing. It is valuable for far-off area interconnections where a little code impression is required and there is restricted organization data transfer capacity. The System utilizes Thing Speak API as a key with a URL to send the information from python IDLE. It can post messages after a ThingSpeak customer is associated with a representative. ThingSpeak has a themebased explanation of the intermediary's messages, so each message needs to comprise specific trouble that the expert will use to deliver the message to dynamic customers. Typically, each message has a payload containing the genuine information to be sent in byte design. ThingSpeak is an information freethinker and the design of the payload relies totally upon the utilization case. On the off chance that you need to send parallel information, literary information, or even undeniable XML or JSON or CSV, it is totally up to the sender. The Sensor center point, Cloud and end User Device all come into the picture while recognizing successively. Above all, data got by the sensor center is shipped off the cloud and besides the end User. In the cloud, getting data is controlled and the assorted task is performed which are inside and out explained as the flowchart.

Fig 2, shows the arrangement of activities in the IAMCS. Exactly when the web affiliation is set up, it will start scrutinizing the boundaries of different sensors. The edge levels for the necessary sensors are set. The sensor data is shipped off the appropriated stockpiling similarly to the end customer. The data can be analyzed down wherever whenever. If the sensor boundaries are more than the cutoff level, by then, the specific alert will be raised and the end client is advised with an alarm. The client can see esteems coming from the sensor hub and distantly control the home apparatuses. At first, Raspberry-pi has been controlled with a $5 \mathrm{~V}$ DC battery. Then, at that point every one of the sensors was interfaced and measured the separate qualities utilizing the regulator, then, at that point, the deliberate qualities and edge esteems are contrasted with answering the water ranchers. 


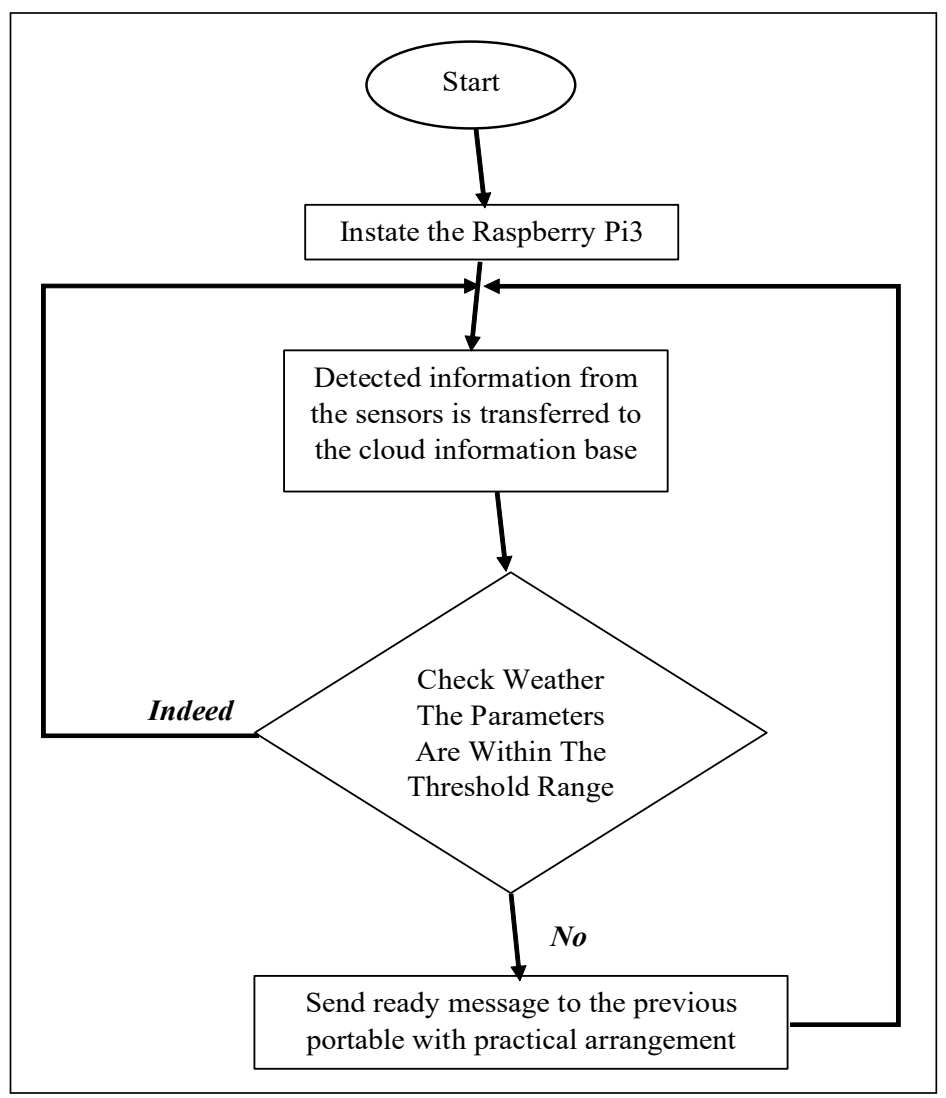

Fig 2: Flowchart for loT based hydroponics checking and control framework.

In a water lake, the proposed framework was carried out and results were acquired utilizing various sensors for 24 hours. Results were gotten with time for fluctuating boundaries of water quality. Fig 3 shows the plot of turbidity shifting with time, the turbidity esteem crosses the edge esteem limit during that specific period and the rancher will get an alarm message to enter new water to the lake. Likewise, Fig 4 and Fig 5 show the variety of pH esteem with time and the variety of temperature with time.

Full circle Delay time (RTD) or The Round-Trip Time (RTT) is that the distance of sum it takings for a piece of information to be sent and the length of your time it takes for partner degree greeting of that information to be gotten. This time delay so comprises the spread occasions between the 2 marks of a side effect. To compute RTT, two python codes run: one distributer and one supporter, for various QoS Publisher, send a period stamp existing apart from everything else it is sent as the message and the endorser prints the time-stamp of the sent just as the got message. The distinction between the got and sent time-stamp to give the RTT. The RTT for various QoS is seen to appear as something else. The RTT is most extreme for QOS-2 and least for QOS-1.

Table 1 comprises different RTTs got during the transmission of a message with various QoS. It additionally shows the normal RTT for each QoS. The table information is last plotted to acquire the diagram that appeared in Fig 6.

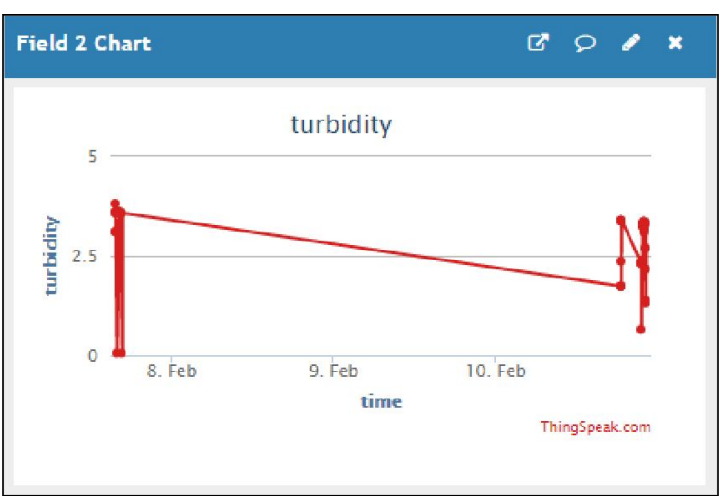

Fig 3: Variation of turbidity with time.

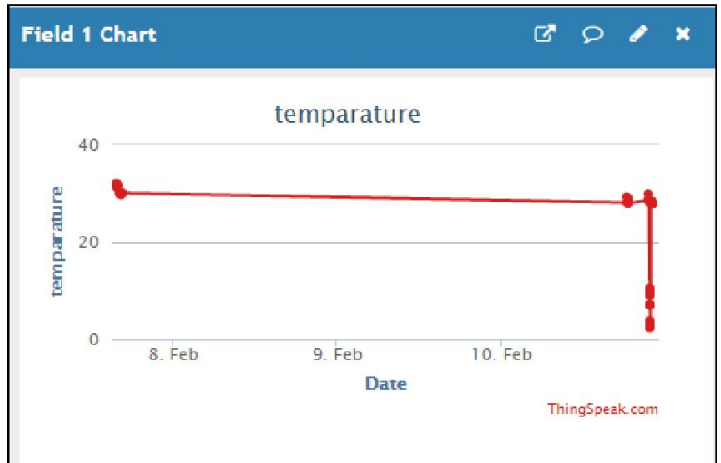

Fig 4: Variation of temperature with time. 
Table 1: Plot of FCD for various QoS.

\begin{tabular}{lccc}
\hline & \multicolumn{3}{c}{ Distinctive QOS } \\
\cline { 2 - 4 } Full Circle Delay Time (FCD) & QOS-1 & QOS-2 & QOS-3 \\
In Seconds & 0.300381 & 0.351937 & 0.987955 \\
& 0.276321 & 0.309702 & 1.11196 \\
& 0.298737 & 0.338293 & 0.913541 \\
& 0.307348 & 0.312028 & 0.906115 \\
& 0.299203 & 0.305806 & 0.925123 \\
& 0.309988 & 0.303237 & 0.922354 \\
& 0.303936 & 0.453029 & 0.980266 \\
& 0.295921 & 0.487394 & 0.930914 \\
& 0.291621 & 0.753097 & 0.866448 \\
& 0.322207 & 0.310773 & 0.913768 \\
Normal FCD & 0.35289 & 0.330946 & 1.000624 \\
\hline
\end{tabular}

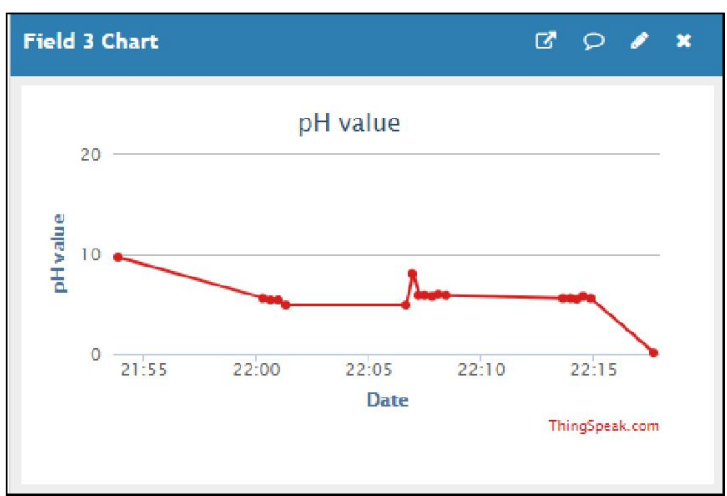

Fig 5: Variation of $\mathrm{pH}$ with time.

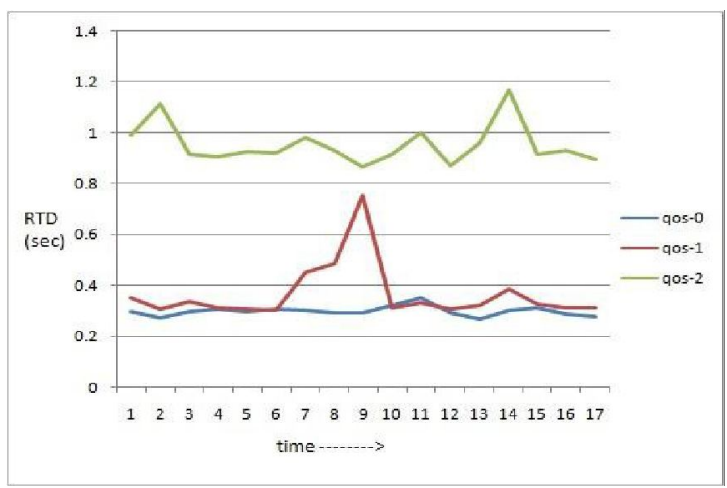

Fig 6: RTT for various QoS.

\section{CONCLUSION}

The procedure executed can work with the water ranchers for the exact and dependable recognition of water boundaries, the undeniable reality that manual testing will take longer and water quality boundaries could change with time It furthermore takes supportive of dynamic measures before any damage was finished. Regardless of the way that the essential expense is high, there will be no additional cost and support whenever it is introduced. Accordingly, the structure executed will arrive at the rancher's for lessening the damage from climatic changes and affirms development and wellbeing for sea-going life. This improves efficiency, helps in improving unfamiliar exchange and builds the GDP of the country. More accumulated data can be assessed using enormous information examination and vital advances can be taken before the water quality boundary crosses the edge esteem range. The water framework robotized utilizing loT diminishes the energy work cost and utilization.

\section{REFERENCES}

Chandanapalli, S.B., Reddy, E.S. and Lakshmi, D.R. (2014). Design and deployment of aqua monitoring system using wireless sensor networks and IAR-Kick. Journal of Aquaculture Research and Development. 5(7).

Chavan, M.S., Patil, M.V.P., Chavan, S., Sana, S. and Shinde, C. (2018). Design and implementation of IOT based real time monitoring system for aquaculture using raspberry pi. International Journal on Recent and Innovation Trends in Computing and Communication. 6(3): 159-161.

Chen, J.H., Sung, W.T. and Lin, G.Y. (2015). October. Automated monitoring system for the fish farm aquaculture environment. In 2015 IEEE International Conference on Systems, Man and Cybernetics IEEE. (pp. 1161-1166).

Deng, C., Gao, Y., Gu, J., Miao, X. and Li, S. (2010). Research on the Growth Model of Aquaculture Organisms Based on Neural Network Expert System. In: 2010 Sixth International Conference on Natural Computation. IEEE. 4: 1812-1815. 
Encinas, C., Ruiz, E., Cortez, J. and Espinoza, A. (2017). April. Design and implementation of a distributed loT system for the monitoring of water quality in aquaculture. In: 2017 Wireless Telecommunications Symposium (WTS) IEEE. (pp. 1-7).

Haron, N.S., Mahamad, M.K.B., Aziz, I.A. and Mehat, M. (2008). August. A System Architecture for Water Quality Monitoring System Using Wired Sensors. In: 2008 International Symposium on Information Technology IEEE. 4: 1-7.

Hongpin, L., Guanglin, L., Weifeng, P., Jie, S. and Quuwei, B. (2015). Real-time remote monitoring system for aquaculture water quality. International Journal of Agricultural and Biological Engineering. 8(6): 136-143.

Israni, Sheetal., Harshal Meharkure. and Parag Yelore. (2015). Application of loT based system for advance agriculture in India. International Journal of Innovative Research in Computer and Communication Engineering 3.11: 1083110837.
Nocheski, S. and Naumoski, A. (2018). Water monitoring loT system for fish farming ponds. Industry 4.0, 3(2): 77-79.

Preetham, K., Mallikarjun, B.C., Umesha, K., Mahesh, F.M. and Neethan, S. (2019). Aquaculture monitoring and control system: An loT based approach. International Journal of Advance Research, Ideas and Innovations in Technology. $5(2)$.

Raju, K.R.S.R. and Varma, G.H.K. (2017). January. Knowledge Based Real Time Monitoring System for Aquaculture using loT. In: 2017 IEEE $7^{\text {th }}$ International Advance Computing Conference (IACC) IEEE. (pp. 318-321).

Simbeye, D.S. and Yang, S.F. (2014). Water quality monitoring and control for aquaculture based on wireless sensor networks. Journal of Networks. 9(4): 840. 